\title{
The Predictive Role of Nephrometry Scores in Evaluating The Effect of Partial Nephrectomy on Postoperative Kidney Functions in T1 Renal Cell Tumors
}

\author{
(D) Müslüm Ergün, (D) Osman Akyüz, (1) Ahmet Hamdi Tefekli \\ Atlas University Faculty of Medicine, Medicine Hospital, Clinic of Urology, Istanbul, Turkey
}

\begin{abstract}
Objective: We aimed at demonstrating the predictive role of different nephrometry scores in evaluating the effect of partial nephrectomy on postoperative kidney functions in patients with T1 kidney tumors.

Materials and Methods: We included 44 patients with clinical stage T1 renal tumors who underwent laparoscopic partial nephrectomy between June 2018 and January 2020. Then, we performed abdominal cross-sectional imaging with computed tomography and magnetic resonance imaging. We recorded the warm ischemia time, operation time, and amount of intraoperative bleeding. The resulting changes were evaluated by determining preoperative, and third month postoperative creatinine and estimated glomerular filtration rates (eGFR). RENAL nephrometry, Preoperative Aspects and Dimensions Used for an Anatomic (PADUA) classificationand Diameter- Axial- Polar (DAP) scores were calculated for each patient. The relationship between a decline in renal function, duration of ischemia and RENAL/PADUA/DAP scores was equally explored to achieve the aim of the study.

Results: The mean age of the patients at the time of surgery was $55.07 \pm 12.92$ years, and the preoperative creatinine and eGFR values were $0.87 \mathrm{mg} / \mathrm{dL}$ and 93.17 $\mathrm{mL} / \mathrm{min}$, respectively. The third month postoperative creatinine and eGFRvalues were determined at $0.96 \mathrm{mg} / \mathrm{dL}$ and $86.52 \mathrm{~mL} / \mathrm{min}$, respectively. The mean RENAL (6.2 \pm 1.4$)$, PADUA (6.8 \pm 1.0$)$, and DAP $(5.57 \pm 1.5)$ scores were also determined. Changes in creatinine and eGFR levels correlated significantly with RENAL/PADUA/ DAP scores and the ischemia time $(p<0.05)$, but not with the operation time, the amount of bleeding, length of hospital stay, tumor size, and location ( $p>0.05)$. Conclusion: Changes in kidney functions and ischemia time correlates significantly withRENAL/PADUA/DAP nephrometry scores.

Keywords: Glomerular filtration rates, creatinine, nephrometry score, partial nephrectomy, renal tumor
\end{abstract}

\section{Introduction}

RENAL cell carcinoma (RCC) constitutes approximately $2 \%$ of all adult cancers, with an increasing annual incidence worldwide (1). Due to the extensive use of radiological imaging methods, the number of patients diagnosed incidentally is also increasing. In addition, technical developments in imaging methods such as dynamic imaging, have facilitated the diagnosis of local enddisease stage tumors in majority of patients (2).

Surgical treatment is an option for patients at the local stage ofRCC and includes partial and radical nephrectomy (3). Partial nephrectomy ( $\mathrm{PN}$ ) has become the standard method in the treatment of T1 a RCC (4). With the application of kidney-sparing surgery, the risks of chronic kidney disease and of cardiovascular disease have greatly decreased (5). Multiple factors can affect kidney function in PN. The presence of comorbidities, the volume of renal parenchyma, and intraoperative ischemia time are listed among these factors (6). Studies have shown that other additional factors related to tumor, patient, and PN techniques may also affect renal functions in the postoperative period $(7,8)$. Nephrometry scores, which are used as preoperative predictors ofrenal function after PN, may be useful in deciding the type of surgery in CT1 kidney masses. For this reason, various nephrometry scores have been developed in order to define masses in patients scheduled for PN and to standardize the choice of the appropriate surgical method $(9,10)$. These developed scoring systems correlate with warm ischemia time and are predictors of postoperative renal functions $(11,12)$. In this study, we aimed at demonstrating the predictive role of different nephrometry scores in our own clinical experience in evaluating the effect of PN on postoperative kidney functions in patients with T1 kidney tumors. 


\section{Materials and Methods}

We analyzed computerized files and written file records of 64 patients who underwent laparoscopic PN (LPN) with an initial diagnosis of renal mass between January 2018 and December 2020 in our institution. Patients with preoperative creatinine values above the reference range $(n=4)$, those with a single kidney $(n=1)$, cases with clinical TNM stage $\geq T 2$ renal tumors $(n=7)$, multiple tumors in the same kidney $(n=2)$, and patients who underwent open PN $(n=6)$ were excluded from the study. The remaining 44 patients were included in the study.

Preoperatively, abdominal cross-sectional imaging (at least one computed tomography and magnetic resonance imaging), which is the gold standard method in the evaluation of renal masses was appliedin 44 patients with clinical stage T1 renal tumors undergoing laparoscopic PN. Complete blood count, blood group determination, and kidney function tests were performed in all patients before surgery. The estimated glomerular filtration rates (eGFRs) before and three months after the operation was estimated using The Simplified Modification of Diet in RENAL Disease Modification of Diet in RENAL Disease equation as follows: (eGFRs $=186 \times$ Serum kreatinin $(\mathrm{mg} / \mathrm{dL})^{-1.154}$ $\times$ age $\left(\right.$ years) ${ }^{-0.203} \times 0.742$ (female) $\times 1.210$ (if Africans) (13). At least one of the abdominal imaging methods (ultrasonography, computer tomography or magnetic resonance imaging) was applied three months after the operation and the patients were followed up thereafter.

Preoperative cross-sectional imaging of the patients was examined for laterality, and size of the lesion, while clinical TNM stage and intrarenal location of the lesion (its exophyticity, proximity to the renal sinus and collecting system) were equally determined. Using preoperative cross-sectional imaging methods, RENAL (lesion size, its exophytic-endophytic nature, proximity to renal sinus, location and distance to the pelvis), Preoperative Aspects and Dimensions Used for an Anatomic (PADUA) (size, exophyticendophytic nature, lateralityof the lesion, its proximity to collecting system and renal sinus, itsdistance to minor calyx) and Diameter- Axial- Polar (DAP) (lesion size, axial distance, polar distance) scores were determined. The patients were divided into three groups according to the RENAL scoring system as having low (4-6 pts), intermediate (7-9 pts) and high (10-12 pts) risks for PN. According to the PADUA scores (which predict the postoperative course after $P N)$, the patients weredivided into low (6-7 pts), intermediate (8-9 pts) and high (10-14 pts) risk categories. According to the predictive DAP scores, the patients were classifiedas low (3-5 pts) and high (6-9 pts) risk groups. During surgery, the duration of artery clamping time (ischemia time), operation time (minute), blood loss (cc), intraoperative complications, requirements for intraoperative transfusion- and hemostatic agent were recorded. Postoperative drainage time, hospital stay, postoperative transfusion need, and postoperative complications were recorded. The relationship between changes in renal functions, duration of ischemia and RENAL/PADUA/DAP scores was examined.

This study was conducted in accordance with the Declaration of Helsinki, and the ethical approval was obtained from our institutional review board (register no: 2018/15-07).

\section{Statistical Analysis}

We analyzed data using the Statistical Package for Social Sciences Statistical Analysis Software version 22.0. Chi-square test was employed for nominal data, t-test for parametric variables, and Mann-Whitney $U$ test for non-parametric variables. Mean \pm standard deviation was used for parametric data, and median \pm distribution width for non-parametric data. A value below $\mathrm{p}<0.05$ was considered statistically significant.

\section{Results}

The mean age of the patients at the time of surgery was $55.07 \pm 12.92$ years, and the female/male ratio was $17 / 27$. Right-side surgery was performed in $47.7 \%$ (21/44) and leftside surgery in $52.3 \%(27 / 44)$ of participants. The mean tumor size was $3.4 \pm 1.35(0.8-6.5) \mathrm{cm}$, and $28(70 \%)$ patients were in clinical stage T1a while $12(30 \%)$ patients in clinical stage T1b (Table 1). Mean RENAL (6.2 \pm 1.4 : range 4-9 pts), PADUA (6.8 \pm 1.0 : range: 6-9 pts), and DAP (5.57 \pm 1.5 : range:4-9 pts) scores were calculated. When the patients were grouped according to the RENAL scoring system, 33 patients (75\%) were in the low-, and 11 patients (25\%) in the intermediate- risk groups. No patients were in the high-risk group. According to the PADUA scoring system, 33 patients (75\%) were in the low-, and $11(25 \%)$ patientsin the high-risk groups. No patient was in the high-risk group as well. According to the DAP scoring system, 25 patients (56.8\%) were in the low-, and19 patients (43.2\%) in the high-risk groups (Table 2).

Preoperative median (range) values for hemoglobin $(13.5 \mathrm{~g} /$ $\mathrm{dL}$ : 8.3-17.1 $\mathrm{g} / \mathrm{dL})$, creatinine $(0.87 \mathrm{mg} / \mathrm{dL}: 0.58-1.39 \mathrm{mg} /$ $\mathrm{dL}$ ), hematocrit (41.5\%: 25.8-51.2 \%) and eGFR $(93.17 \mathrm{~mL} /$ min: $58.33-136.81 \mathrm{~mL} / \mathrm{min}$ ) were obtained accordingly. Postoperative third month median (range) creatinine $(0.96 \mathrm{mg} /$ $\mathrm{dL}$ : 0.6-1.66 mg/dL) and eGFR (86.52 mL/min: 46.5-119.26 $\mathrm{mL} / \mathrm{min}$ ) values were also calculated. A significant difference was found between preoperative andpostoperative creatinine and eGFR values of the patients $(p<0.05)$ (Table 3$)$. Changes in eGFR showed a significant correlation with RENAL/PADUA/DAP scores and duration ofischemia $(p=0.045, p=0.037, p=0.041$, and $p=0.003$, respectively); Operation time, amount ofbleeding, hospital stay, tumor diameter, and location did not show any significant correlation $(p=0.212, p=0.880, p=0.620, p=0.078$, $\mathrm{p}=0.091$, and $\mathrm{p}=0.081$, respectively) (Table 4 ).

The mean operation time was $130.23 \pm 13.97$ (100-160) minutes and the mean amount of blood loss was 290.34 \pm 107.58 (110$600)$ cc. Warm ischemia was applied to the kidney during tumor resection in all patients who underwent PN. Arterial clamping, andselective arterial clamping were applied in $81.8 \%$, and $18.2 \%$ of the patients who underwent PN with warm ischemia, respectively. Hemostatic agents were used in the tumor bed in 88.6\% (39/44) of the patients who underwent PN. Considering the intraoperative and postoperative complications, there were no complications except the requirement for blood transfusion in three patients. Blood transfusion was required intraoperatively in $4.5 \%(2 / 44)$ of the patients, and in $2.3 \%(1 / 144)$ of cases during the postoperative follow-up.No case of postoperative mortality was recorded. 


\begin{tabular}{|c|c|c|}
\hline Variables & \multicolumn{2}{|c|}{ Mean \pm SD or number, ratio } \\
\hline Age (years) & \multicolumn{2}{|c|}{$55.07 \pm 12.92(28-85)$} \\
\hline Gender (M/F) & \multicolumn{2}{|c|}{$27 / 17(44)$} \\
\hline Indication of RPN & \multicolumn{2}{|l|}{44} \\
\hline Elective & \multicolumn{2}{|l|}{44} \\
\hline Mandatory & \multicolumn{2}{|l|}{0} \\
\hline Laterality (right/left) & \multicolumn{2}{|c|}{$21 / 23(44)$} \\
\hline Tumor size, cm & \multicolumn{2}{|c|}{$3.4 \pm 1.35(0.8-6.5)$} \\
\hline \multicolumn{3}{|l|}{ Tumor classification } \\
\hline Endophytic & \multicolumn{2}{|c|}{$12(27 . \% 7)$} \\
\hline Exophytic & \multicolumn{2}{|c|}{$32(72.3 \%)$} \\
\hline Central & \multicolumn{2}{|l|}{0} \\
\hline \multicolumn{3}{|l|}{ Tumor location } \\
\hline Upper pole & \multicolumn{2}{|c|}{$9(20.5 \%)$} \\
\hline Midpole & \multicolumn{2}{|c|}{$9(20.5 \%)$} \\
\hline Lower pole & \multicolumn{2}{|c|}{$22(50 \%)$} \\
\hline Anterior & \multicolumn{2}{|c|}{$1(2.3 \%)$} \\
\hline Posterior & \multicolumn{2}{|c|}{$3(6.8 \%)$} \\
\hline OpeOperative time, min & \multicolumn{2}{|c|}{$130.23 \pm 13.97(100-160)$} \\
\hline Warm ischemia time, min & \multicolumn{2}{|c|}{$20.07 \pm 4.38(10-34)$} \\
\hline Average blood loss, $\mathrm{mL}$ & \multicolumn{2}{|c|}{$290.34 \pm 107.58(110-600)$} \\
\hline Conversion to open surgery & \multicolumn{2}{|c|}{$0 / 44$} \\
\hline Transfusion & \multicolumn{2}{|c|}{$3 / 44$} \\
\hline Ürinoma & $0 / 4$ & \\
\hline Perinephritic hematoma & $0 / 4$ & \\
\hline Drain dwell time & 2.02 & $\pm 0.89(0-5)$ \\
\hline Hospital stay, days & $3.8 \pm$ & $0.79(3-6)$ \\
\hline Pathology & & \\
\hline Malignant & 40( & $90.9 \%)$ \\
\hline Benign & $4(9$ & $1 \%)$ \\
\hline Positive surgical margin & $1 / 4$ & $(2.27 \%)$ \\
\hline RCC type & & \\
\hline Clear cell & 26 & $59.1 \%)$ \\
\hline Papillary & 8 & $(18.2 \%)$ \\
\hline Chromophobe & 2 & $(4.5 \%)$ \\
\hline Chromophobe + eosinophilic & 1 & $(2.3 \%)$ \\
\hline Mucinous tubular & 2 & $(4.5 \%)$ \\
\hline İnvasive squamous & 1 & $(2.3 \%)$ \\
\hline RCC grades & & \\
\hline 1 & 12 & $(30 \%)$ \\
\hline 2 & 26 & $(65 \%)$ \\
\hline 3 & 2 & $(5 \%)$ \\
\hline 4 & 0 & $(0 \%)$ \\
\hline RCC stage & & \\
\hline PT1a & 28 & $(7 \% 0)$ \\
\hline PT1b & 12 & $(30 \%)$ \\
\hline Benign & 4 & $(9.1 \%)$ \\
\hline Oncocytoma & 3 & $(6.8 \%)$ \\
\hline Atypical lipomatous tumor & 1 & $(2.3 \%)$ \\
\hline RCC: RENAL cell carcinoma, SD: & viation & , M: Male, F: Female \\
\hline
\end{tabular}

\begin{tabular}{|c|c|c|c|}
\hline \multicolumn{2}{|l|}{ Scores } & \multicolumn{2}{|l|}{ n - mean scores } \\
\hline \multirow{3}{*}{ RENAL } & Low & $33 / 44(75 \%)$ & \multirow{2}{*}{$6.2 \pm 1.4(4-9)$} \\
\hline & Intermediate & $11 / 44(25 \%)$ & \\
\hline & High & $0 / 44(0 \%)$ & \\
\hline \multirow{3}{*}{ PADUA } & Low & $33 / 44(75 \%)$ & \multirow{2}{*}{$6.8 \pm 1.0(6-9)$} \\
\hline & Intermediate & $11 / 44(25 \%)$ & \\
\hline & High & $0 / 44(0 \%)$ & \\
\hline \multirow{2}{*}{ DAP } & Low & $25 / 44(56 \% .8)$ & \multirow{2}{*}{$5.57 \pm 1.5(4-9)$} \\
\hline & High & $19 / 44(43 \% .2)$ & \\
\hline
\end{tabular}

Table 3. Changes in creatinine, and eGFR values of the patients during follow-up period relative to preoperative values

\begin{tabular}{|l|l|}
\hline & $\begin{array}{l}\text { PreoperativePostoperative } \\
\text { (mean } \pm \text { SD, max-min) (mean } \pm \text { SD, max-min) } \\
\text { p-value }\end{array}$ \\
\hline Creatinine (mg/dL) & $\begin{array}{l}0.87 \pm 0.17(0.58-1.39) 0.96 \pm 0.20 \\
(0.6-1.66) 0.032\end{array}$ \\
\hline eGFR (mL/min) & $\begin{array}{l}93.17 \pm 15.66(58.33-136.81) ~ 86.52 \pm 16.81 \\
(46.5-119.26) 0.04\end{array}$ \\
\hline $\begin{array}{l}\text { eGFR: Estimated glomerular filtration rates, SD: Standard deviation, min: } \\
\text { Minimum, max: Maximum }\end{array}$ \\
\hline
\end{tabular}

Table 4. Statistical comparison between intraoperative, and postoperative findings of the participants according to changes in eGFR, and creatinine values

\begin{tabular}{|l|l|l|}
\hline & $\begin{array}{l}\text { Changes in egfr } \\
\text { p-values }\end{array}$ & $\begin{array}{l}\text { Changes in } \\
\text { creatinine } \\
\text { p-values }\end{array}$ \\
\hline Amount of bleeding & 0.880 & 0.856 \\
\hline Drain dwell time (days) & 0.212 & 0.308 \\
\hline Hospital stay (days) & 0.620 & 0.452 \\
\hline RENAL score & $0.045^{*}$ & $0.046^{*}$ \\
\hline PADUA score & $0.037^{*}$ & $0.040^{*}$ \\
\hline DAP score & $0.041^{*}$ & $0.039^{*}$ \\
\hline Tumor diameter & 0.078 & 0.069 \\
\hline Exophytic renal tumor & 0.091 & 0.071 \\
\hline Endophytic renal tumor & 0.080 & 0.066 \\
\hline Ischemia time & $0.003^{*}$ & $0.013^{*}$ \\
\hline Intraoperative transfusion & 0.174 & 0.584 \\
\hline Perioperative transfusion & 0.198 & 0.108 \\
\hline $\begin{array}{l}\text { *: Statistically significant, eGFR: Estimated glomerular filtration rates, } \\
\text { PADUA: Preoperative Aspects and Dimensions Used for an Anatomic, DAP: } \\
\text { Diameter- Axial- Polar }\end{array}$ \\
\hline
\end{tabular}

Histopathological evaluation of the patients, revealedthat 40 patients (90.9\%) had malignant tumors and $4(9.1 \%)$ had benign lesions. Malignant pathologies were reported as clear cell RCC in 26 patients (59.1\%), papillary RCC in 8 patients (18.2\%) and other RCCs in $6(13.6 \%)$ patients. Three of four patients with benign pathology had oncocytomas. Surgical margin positivity was seen in one patient. Table 1 displays 
data regarding histological subtypes and RCC grades in the pathology specimens of the patients. Mean drain dwell time was $2.02 \pm 0.89$ (0-5) days, and the mean hospital stay was $3.8 \pm 0.79$ (3-6) days.

\section{Discussion}

In experienced centers, LPN has proven to be an alternative method to open surgery owing to its peroperative and oncological results in both stage $1 \mathrm{a}$ and stage $1 \mathrm{~b}$ tumors $(14,15)$. The fact that LPN is technically more complicated, needing advanced laparoscopic skills and the long learning curve makes it difficult to perform LPN outside centers with a heavy patient load. In a multicenter study performed in patients with solitary kidney, it was stated that the warm ischemia time should be limited to 20-35 minutes in order to prevent irreversible kidney damage (16). In our series, the median warm ischemia time was 20.07 (10-34) minutes, and the median operative time was 130 (100-160) minutes, consistent with the literature. Severalstudies have indicated that the use of intravenous mannitol during PN, the total operative time, amount of blood loss and additional comorbidities may affect postoperative eGFR, while others have stated that these parameters will not necessarily affect postoperative eGFR $(17,18)$.

One of the main goals of PN surgery is to preserve as much functional kidney tissue as possible as well as preserving kidney functions in the shortest possible warm is chemia period. In our study, arterial clamping, and selective arterial clamping were applied in $81.8 \%$, and $18.2 \%$ of the patients who underwent LPN under warm ischemia, respectively, and a significant relationship was found between the ischemia time, a decrease in the eGFR and an increase in creatinine levels of the patients in the postoperative third month. Shah et al. (19) examined changes ineGFR values in the follow-up period of 315 patients who underwent elective ischemic and zero-ischemic PN. They showed that although there was a significant decrease in eGFR in the early postoperative period in patients with ischemic PN, this difference disappeared at the $6^{\text {th }}$ postoperative month. Mir et al. (20) reported demonstrate no relationship between ischemia time and preserved renal function. Porpiglia et al. (21) employed renal scintigraphy at the third postoperative month to evaluate the patients they applied PN, and reported lack of any significant difference in terms of loss of renal function between ischemicand zero-ischemic groups. In another study, it was reported that postoperative function was worst in patients with poor basal kidney function (22).

In our study, postoperative changes in eGFR and creatinine values showed a significant correlation with all nephrometry score groups. Recently, Selvi and Başar (23) showed that RENAL and PADUA scores were more important predictors of decline in eGFR than tumor size and stage. Considering the lack of highrisk patients in our study according to assessments performed with RENAL and PADUA scoring systems, we anticipate that this correlation may be more pronounced in patients with highrisk scores. In a series of 188 patients, Borgmann et al. (11) demonstrateda correlation between RENAL, PADUA, and DAP scores with ischemia time. Similarly, in a series of 101 patients, Okhunov et al. (12) revealed thatRENAL and PADUA scores correlated positively with warm ischemia time. As a matter of fact, in our study, durationofischemia demonstrated a significant correlation with the RENAL, PADUA, and DAP scores.

No significant correlation was found with the operation time, tumor diameter, length of stay, amount of bleeding, endophytic, and exophytic nature of the tumor and changes in GFR. Although some studies demonstrate changes in eGFR depending on the exophytic or endophytic nature of the tumor, we believe that this is related to the experience of the surgical team. In our series, we think that the surgical team consisting of the same experienced urologists was an effective factor on our successful outcomes. As a matter of fact, Kim et al. (24) reported that size, location, type of the tumor and duration of ischemia had no effect on postoperative renal functions.

\section{Study Limitations}

Our study has some limitations that should be considered. First, the use of eGFR instead of direct GFR measurments will create an error in analysis. Second, low number of patients and failure to follow-up our patients for sufficiently longer period can be counted among other limitations.

\section{Conclusion}

From our results, preoperative nephrometry scores are as important as surgical factors in determining the possibility of decreased kidney function in cT1 kidney tumors. Considering the positive correlation between duration ofischemia and renal functions, the importance of surgical experience comes to play. In our study, a significant correlation existed between renal functions, duration ofischemia and RENAL/PADUA/DAP scores. This result indicates the importance of nephrometry scores in predicting the postoperative decline in renal function. Still, more advanced and validated predictive nomograms are needed to predict short- and long-term kidney function.

\section{Acknowledgements}

Publication: The results of the study were not published in full or in part in form of abstracts.

Contribution: Assoc. Prof. Kerem Taken, Yüzüncü Yıl University Faculty of Medicine.

Conflict of Interest: No conflict of interest was declared by the authors.

Financial Disclosure: The authors declared that this study received no financial support.

\section{Ethics}

Ethics Committee Approval: This study was conducted in accordance with the Declaration of Helsinki, and the ethical approval was obtained from our institutional review board (register no: 2018/15-07).

Informed Consent: Retrospective study.

Peer-review: Externally peer-reviewed.

\section{Authorship Contributions}

Concept: M.E., O.A., A.H.T., Design: O.A., M.E., A.H.T., Data Collection or Processing: M.E., O.A., A.H.T., Analysis or 
Interpretation: M.E., O.A., A.H.T., Literature Search: M.E., O.A., A.H.T., Writing: M.E., O.A., A.H.T.

\section{References}

1. Ferlay J, Soerjomataram I, Dikshit R, et al. Cancer incidence and mortality worldwide: sources, methods and major patterns in GLOBOCAN 2012. Int J Cancer 2015;136:E359-E86. doi: 10.1002/ ijc.29210.

2. Siegel RL, Miller KD, Jemal A. Cancer statistics, 2018. CA Cancer J Clin 2018;68:7-30.

3. Pierorazio PM, Johnson MH, Patel HD, et al. Management of renal masses and localized renal cancer: systematic review and metaanalysis. J Urol 2016;196:989-999.

4. Ljungberg B, Hanbury DC, Kuczyk MA, et al. Renal cell carcinoma guideline. Eur Urol 2007;51:1502-1510.

5. Patel HD, Pierorazio PM, Johnson $\mathrm{MH}$, et al. Renal functional outcomes after surgery, ablation, and active surveillance of localized renal tumors: a systematic review and meta-analysis. Clin J Am Soc Nephrol 2017;12:1057-1069.

6. Kim SP, Thompson RH, Boorjian SA, et al. Comparative effectiveness for survival and renal function of partial and radical nephrectomy for localized renal tumors: a systematic review and meta-analysis. J Urol 2012;188:51-57.

7. Bhindi $B$, Lohse $C M$, Schulte $\mathrm{PJ}$, et al. predicting renal function outcomes after partial and radical nephrectomy. Eur Urol 2019;75:766-772.

8. Shum CF, Bahler CD, Cary C, et al. Preoperative nomograms for predicting renal function at 1 year after partial nephrectomy. J Endourol 2017;31:711-718.

9. Ficarra V, Novara G, Secco S, et al. Preoperative aspects and dimensions used for an anatomical (PADUA) classification of renal tumours in patients who are candidates for nephron-sparing surgery. Eur Urol 2009;56:786-793.

10. Simmons $M N$, Hillyer SP, Lee $B H$, et al. Diameter-axial-polar nephrometry: integration and optimization of R.E.N.A.L. and centrality index scoring systems. J Urol 2012;188:384-390.

11. Borgmann $H$, Reiss AK, Kurosch $M$, et al. R.E.N.A.L. score outperforms PADUA score, C-Index and DAP score for outcome prediction of nephron sparing surgery in a selected cohort. J Urol 2016;196:664-671.

12. Okhunov Z, Rais-Bahrami S, George AK, et al. The comparison of three renal tumor scoring systems: C-Index, P.A.D.U.A., and R.E.N.A.L. nephrometry scores. J Endourol 2011;25:1921-1924.
13. Levey AS, Coresh J, Greene T, et al. Chronic Kidney Disease Epidemiology Collaboration. Using standardized serum creatinine values in the modification of diet in renal disease study equation for estimating glomerular filtration rate. Ann Intern Med 2006; 145:247-254.

14. Simmons MN, Weight C], Gill IS. Laparoscopic radical versus partial nephrectomy for tumors $>4 \mathrm{~cm}$ : intermediate-term oncologic and functional outcomes. Urology 2009;73:1077-1082.

15. Van Poppel H, Da Pozzo L, Albrecht W, et al. A prospective, randomised EORTC intergroup phase 3 study comparing the oncologic outcome of elective nephron-sparing surgery and radical nephrectomy for low-stage renal cell carcinoma. Eur Urol 2011;59:543-552.

16. Thompson RH, Frank I, Lohse CM, et al. The impact of ischemia time during open nephron sparing surgery on solitary kidneys: a multiinstitutional study. J Urol 2007;177:471-476.

17. Breau RH, Cagiannos I, Knoll G, et al. Renal hypothermia during partial nephrectomy for patients with renal tumours: a randomised controlled clinical trial protocol. BMJ Open 2019;9:e025662.

18. Klingler MJ, Babitz SK, Kutikov A, et al. Assessment of volume preservation performed before or after partial nephrectomy accurately predicts postoperative renal function: Results from a prospective multicenter study. Urol Oncol 2019;37:33-39.

19. Shah PH, George AK, Moreira DM, et al. To clamp or not to clamp? Long-term functional outcomes for elective off-clamp laparoscopic partial nephrectomy. BJU Int 2016;117:293-299.

20. Mir MC, Campbell RA, Sharma N, et al. Parenchymal volume preservation and ischemia during partial nephrectomy: functional and volumetric analysis. Urology 2013;82:263-268.

21. Porpiglia F, Bertolo R, Amparore D, et al. Evaluation of functional outcomes after laparoscopic partial nephrectomy using renal scintigraphy: clamped vs clampless technique. BJU Int 2015;115:606-612.

22. Li Y, Zhou L, Bian T, et al. The zero ischemia index (ZII): a novel criterion for predicting complexity and outcomes of off-clamp partial nephrectomy. World J Urol 2017;35:1095-1102.

23. Selvi I, Başar H. Predictive factors for postoperative decline in renal functions following partial nephrectomy: preliminary results. J Urol Surg 2020;7:92-102.

24. Kim SH, Kang KM, Yu A, et al. A study of relationship of atheroembolic risk factors with postoperative recovery in renal function after partial nephrectomy in patients staged T1-2 renal cell carcinoma during median 4 year follow-up. Cancer Res Treat 2016;48:288-296. 